\title{
UV luminescence in Gd-doped silica and phosphosilicate optical fibers
}

\author{
Y. Wang ${ }^{1}$, J. He ${ }^{1}$, P. Barua ${ }^{1}$, N. Chiodini ${ }^{2}$, S. Steigenberger ${ }^{3}$, M. I. M. Abdul Khudus ${ }^{1,4}$, J.K. Sahu ${ }^{1}$, M. Beresna ${ }^{1}$ \\ and G. Brambilla ${ }^{1}$ \\ 1. Optoelectronics Research Centre, University of Southampton, Southampton, SO17 1BJ, United Kingdom \\ 2. Dipartimento di Scienza dei Materiali, Università di Milano Bicocca, 20126 Milan, Italy \\ 3. National Oceanography Centre, Southampton, SO14 3ZH, United Kingdom \\ 4. Photonics Research Centre, Department of Physics, Faculty of Science, University of Malaya, 50603 Kuala Lumpur, Malaysia
}

\begin{abstract}
Gd-doped silica and phosphosilicate fibers were pulled from preforms fabricated using the rod-in-tube technique and the solution doping technique, respectively. Ultraviolet (UV)B luminescence from trivalent $\mathrm{Gd}$ at around $312 \mathrm{~nm}$ given by transition from first excited state to ground state were observed under deep UV excitations.
\end{abstract}

Keywords_-gadolinium; photoluminescence; ultraviolet; fiber

\section{INTRODUCTION}

Optical fiber laser sources exhibit unsurpassed beam quality and power handling capabilities. Silica fibers are the favorite hosts for lasing ions as they tend to have high temporal stability and mechanical strength as well as strong chemical and radiation resistance. They are compatible with existing silica-based optical fiber components and can be transparent in the ultraviolet (UV) [1]. Up to date numerous fiber lasers operating in the near infrared and visible have been demonstrated, based on rare earths such as $\mathrm{Yb}^{3+}, \mathrm{Er}^{3+}, \mathrm{Ce}^{3+}$ and $\mathrm{Tm}^{3+}$. Short wavelengths have been generated through upconversion process, such as $\mathrm{LiYF}_{4}:$ Er operating at $551 \mathrm{~nm} \mathrm{[2],}$ $\mathrm{Ho}^{3+}$ fluorozirconate fiber laser emitting between 540 and $553 \mathrm{~nm}$ [3], $\mathrm{Pr}^{3+} / \mathrm{Yb}^{3+}$ codoped ZBLAN fiber lasers tunable in the red, orange, green, and blue wavelength range [4], and $\mathrm{LiYF}_{4}: \mathrm{Tm}$ fluorescing near $350 \mathrm{~nm}$ and $450 \mathrm{~nm}$ and weaker features at wavelengths down to $288 \mathrm{~nm}$ under avalanche pumping [5]. Extension to the UV would benefit medical and industrial applications, where, at present, gas lasers, lamps, diodes or sources based on nonlinear harmonic generations are mostly used [6]-[9]. Here, we investigated the UV-B luminescence emissions from silica fibers single doped with trivalent gadolinium ions, $\mathrm{Gd}^{3+}$, with an energy gap of $\sim 32000$ $\mathrm{cm}^{-1}$ between the ground state $\left({ }^{8} \mathrm{~S}_{7 / 2}\right)$. and the first excited level $\left({ }^{6} \mathrm{P}_{7 / 2}\right)$ (Fig. 1$)$.

\section{SAMPLE PREPARATION}

Two types of fiber core host materials, i.e., phosphosilicate and pure silica, were investigated. The phosphosilicate sample had a pure silica cladding and a Gd doped phosphosilicate core fabricated using the modified chemical vapor technique (MCVD), with vapor-phase $\mathrm{SiCl}_{4}$ and $\mathrm{POCl}_{3}$ precursors, and the solution doping technique [12], with $\mathrm{GdCl}_{3} \cdot 6 \mathrm{H}_{2} \mathrm{O}$ in a methanol solution. The dip at the center of fiber index profile (Fig.2, black) has been attributed to the evaporation of $\mathrm{P}_{2} \mathrm{O}_{5}$ during soot consolidation and tube collapse. The average $\mathrm{Gd}^{3+}$ concentration in the core, measured using energy-dispersive $\mathrm{X}$ ray spectroscopy (EDX), was $\sim 1170 \mathrm{ppm}$. The preform was pulled into a fiber at temperature above $2000{ }^{\circ} \mathrm{C}$. The fiber cladding and core diameters were $5.8 \mu \mathrm{m}$ and $125 \mu \mathrm{m}$, respectively.

The $\mathrm{Gd}^{3+}$ doped silica sample had a silica core fabricated by sol-gel [13] and a fluorosilicate cladding added via rod-intube technique. The fiber sample has a top-hat index profile (Fig.2 blue). Characterization of the sol-gel rod had been previously presented elsewhere [14]. The rod without the cladding was pulled into fiber canes for the PL measurements.

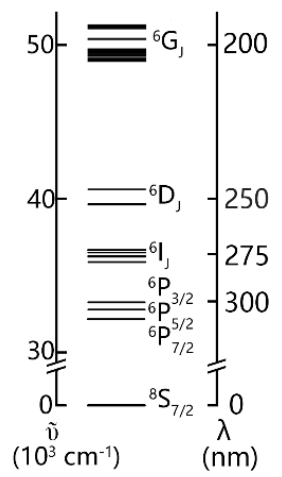

Fig. 1. Schematic of the $\mathrm{Gd}^{3+}$ energy levels (adapted from reference [10] and [11]). $\tilde{v}$ and $\lambda$ are the wavenumbers and wavelengths of the photons emitted in the transitions to the ground level.

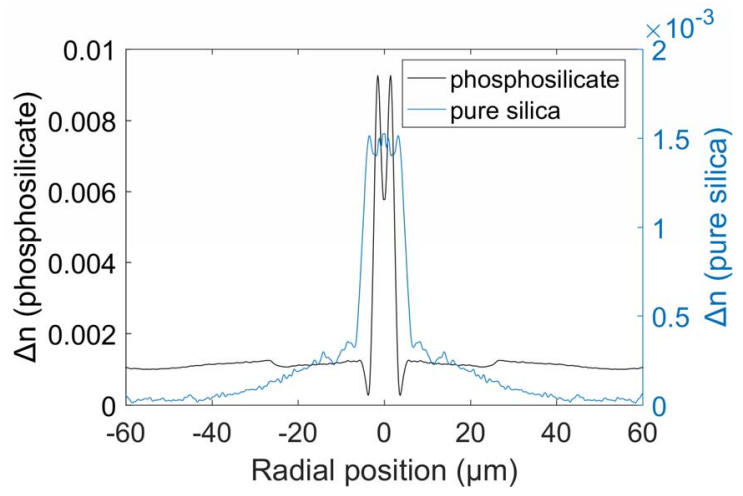

Fig. 2. Refractive index profiles of the Gd-doped optical fibers with phosphosilicate and pure silica cores. 


\section{UV ABSORPTION SPECTRA}

Absorption associated to $\mathrm{Gd}^{3+}$ doping in the phosphosilicate fiber (Fig. 3) was investigated by pumping it with a broadband deuterium lamp source (BDS130, BWTEK). The propagation loss measured via the cutback method shows relatively narrow peaks at the wavelengths $\lambda=301.4 \mathrm{~nm}$, $306.3 \mathrm{~nm}$ and $311.9 \mathrm{~nm}$, attributed to the $\mathrm{Gd}^{3+}$ transitions from the fundamental $\left({ }^{8} \mathrm{~S}_{7 / 2}\right)$ to the ${ }^{6} \mathrm{P}_{\mathrm{J}}$ multiplet levels. High losses at short wavelengths are mostly related to defects [15] and to the small phosphosilicate electronic bandgap [16]. Since luminescence from oxygen-deficient centers (ODCs) was observed in a previous work [17], an attempt to reduce loss by reducing the concentration of ODCs was carried out by placing phosphosilicate optical fiber samples in an oxygen loading cell at 200 bar and $60^{\circ} \mathrm{C}$ for one month. To avoid overlaps with the Gd absorptions associated to the ${ }^{8} \mathrm{~S}_{7 / 2} \rightarrow{ }^{6} \mathrm{D}_{\mathrm{J}}$ transition, changes in the absorption related to oxygen loading were monitored by measuring the propagation loss at $\lambda=260 \mathrm{~nm}$. The cut-back measurements are presented in Fig. 4 and show a $50 \%$ reduction from $0.5 \mathrm{~dB} / \mathrm{cm}$ to $0.23 \mathrm{~dB} / \mathrm{cm}$. Additionally, nonbridging oxygen hole center (NBOHC), another type of defect,

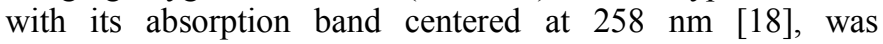
investigated by detecting its red luminescence using a spectrofluorometer (Fluorolog-3, Horiba), and it turned out to be negligible in both Gd-doped phosphosilicate and pure silica fiber samples. Further investigation and minimization of loss at short wavelengths would be required for future work.

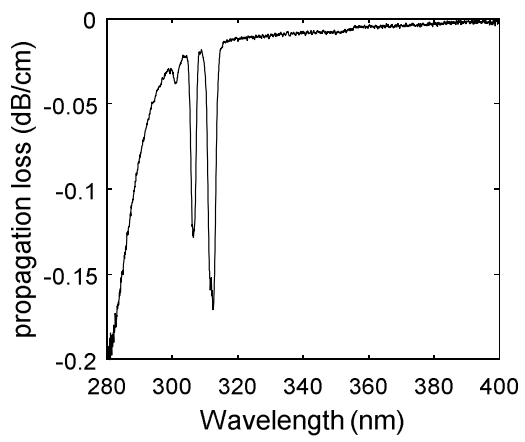

Fig. 3. Propagation loss of the phosphosilicate optical fiber.

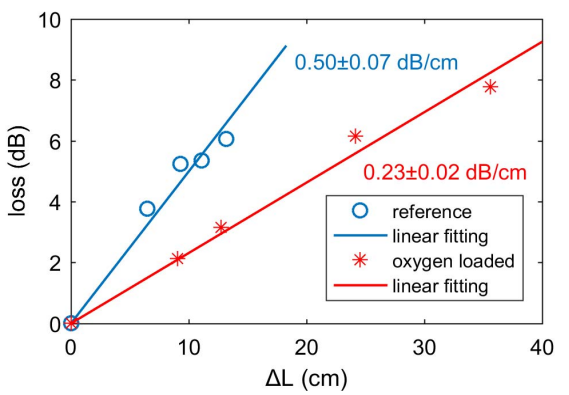

Fig. 4. Cut-back measurements used to determine the propagation loss at $\lambda=260 \mathrm{~nm}$ before and after oxygen loading.

\section{PhotoluminesCenCE}

A Horiba Fluorolog-3 spectrofluorometer equipped with a Xenon excitation lamp were used to record the photoluminescence (PL) and the photoluminescence excitation (PLE) spectra of a disk cut from the phosphosilicate preform, a bundle of phosphosilicate fibers and a stack of silica fiber canes. To improve the signal-to-noise ratio $(\mathrm{S} / \mathrm{N})$, light was incident on the sample sides, which have the highest core to cladding aspect ratio.

UV-B emission originating from the $\mathrm{Gd}^{3+}{ }^{6} \mathrm{P}_{7 / 2} \rightarrow{ }^{8} \mathrm{~S}_{7 / 2}$ transition was observed at $\lambda=311.7 \mathrm{~nm}$ from the phosphosilicate preform disk. PLE peaks were recorded at $\lambda_{\mathrm{ex}}=243.7 \mathrm{~nm}, 245.7 \mathrm{~nm}$ and $251.9 \mathrm{~nm}$, when pumping into the ${ }^{6} \mathrm{D}_{\mathrm{J}}$ levels; at $\lambda_{\mathrm{ex}}=272.6 \mathrm{~nm}, 275.2 \mathrm{~nm}$ and $278.3 \mathrm{~nm}$, when pumping into the ${ }^{6} \mathrm{I}_{\mathrm{J}}$ levels; and at $\lambda_{\mathrm{ex}}=304.0 \mathrm{~nm}$, when pumping into the ${ }^{6} \mathrm{P}_{5 / 2}$ levels, respectively (Fig. 5, red).

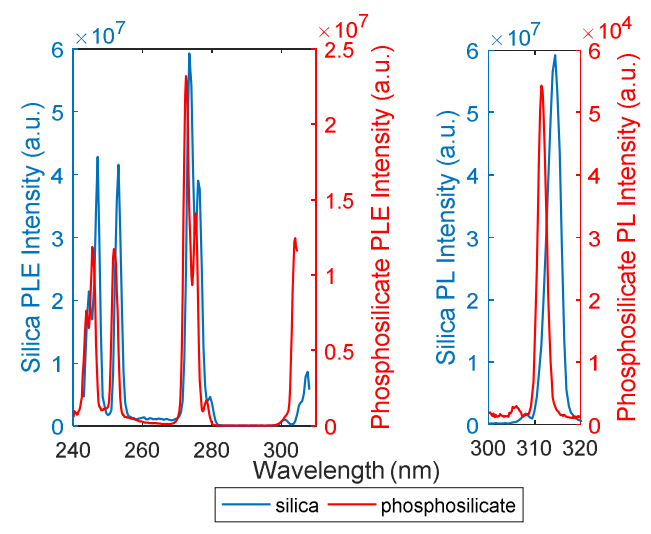

Fig. 5. PL and PLE spectra of Gd-doped samples with pure silica $\left(\lambda_{\mathrm{ex}} \sim 274 \mathrm{~nm}, \lambda_{\mathrm{em}} \sim 314 \mathrm{~nm}\right)$ and phosphosilicate $\left(\lambda_{\mathrm{ex}} \sim 272.5 \mathrm{~nm}, \lambda_{\mathrm{em}} \sim 312 \mathrm{~nm}\right)$ cores.

These transitions feature a spin change and show linewidths of the order of $\Delta \lambda \sim 2.5 \mathrm{~nm}$ (FWHM) in the PL spectrum. Fig. 6 presents a comparison of the PL and PLE spectra recorded from the $\mathrm{Gd}^{3+}$-doped phosphosilicate optical fiber and from the fiber preform used to fabricate it: a comparison suggests that the thermal treatment during the fiber pulling process did not affect the position of the PLE peak wavelengths.

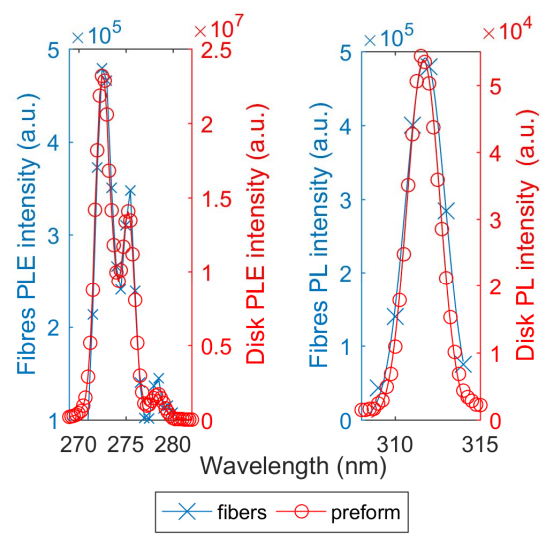

Fig. 6. PL $\left(\lambda_{\mathrm{ex}} \sim 272.5 \mathrm{~nm}\right)$ and PLE $\left(\lambda_{\mathrm{em}} \sim 312 \mathrm{~nm}\right)$ spectra of phosphosilicate preform disk and fibers.

Similar results were recorded from the $\mathrm{Gd}^{3+}$-doped silica fiber cane, with a wavelength shift of $\Delta \lambda \sim 1.5 \mathrm{~nm}$ with respect 
to the phosphosilicate samples (Fig. 5, blue): the peak emission wavelength $(314 \mathrm{~nm})$ was unchanged when the preform rod was pulled into an optical fiber [14].

\section{CONCLUSION}

UV-B PL emissions at $\lambda=311.7 \mathrm{~nm}$ and $314 \mathrm{~nm}$ were recorded in $\mathrm{Gd}^{3+}$-doped phosphosilicate and silica fibers, respectively. The absorption and emission wavelengths were not affected by thermal treatments during fiber pulling at a high temperature. A spectral shift of $\Delta \lambda \sim 1.5 \mathrm{~nm}$ caused by the difference in the host materials was recorded. Absorption at short wavelengths was reduced via oxygen loading process. To work towards laser operation, in addition to a high power pump source, future work will need 1) to perform a quantitative measurement of absorption cross-sections, 2) to manufacture the laser cavity possibly by writing mirrors directly into the active fiber core using femtosecond laser irradiation and 3) to further improve the material transparency in order to achieve a large gain at the Gd fluorescence wavelengths.

\section{ACKNOWLEDGMENT}

The authors would like to thank Neil Sessions at the ORC for support in the cleanroom and holder fabrication. The authors gratefully acknowledge financial support from the UK Engineering and Physical Sciences Research Council through the grant EP/L01243X/1.

\section{REFERENCES}

[1] Fiberguide Industries Limited. (2012, Sep). Solarization Resistant UV Fiber [Online]. Available: https://www.fiberguide.com/wpcontent/uploads/2012/09/Solarguide 090712.pdf

[2] R. A. McFarlane, "High-power visible upconversion laser," Opt. Lett., vol. 16, no. 18, p. 1397, Sep. 1991.

[3] J. Y. Allain, M. Monerie, and H. Poignant, "Room temperature CW tunable green upconversion holmium fibre laser," Electron. Lett., vol. 26, no. 4, p. 261, 1990.

[4] P. Xie and T. R. Gosnell, "Room-temperature upconversion fiber laser tunable in the red, orange, green, and blue spectral regions," Opt. Lett., vol. 20, no. 9, p. 1014, May 1995.
[5] A. W. Kueny, W. E. Case, and M. E. Koch, "Infrared-to-ultraviolet photon-avalanche-pumped upconversion in Tm:LiYF 4 ," J. Opt. Soc. Am. $B$, vol. 10, no. 10, p. 1834, Oct. 1993.

[6] K. Song, M. Mohseni, and F. Taghipour, "Application of ultraviolet light-emitting diodes (UV-LEDs) for water disinfection: A review," Water Res., vol. 94, pp. 341-349, 2016.

[7] J. M. Hoffman, A. K. Hays, and G. C. Tisone, "High power uv noblegas-halide laserf," Appl. Phys. Lett., vol. 28, no. 9, pp. 538-539, 1976.

[8] H. M. Pask, P. Dekker, R. P. Mildren, D. J. Spence, and J. A. Piper, "Wavelength-versatile visible and UV sources based on crystalline Raman lasers," Prog. Quantum Electron., vol. 32, no. 3-4, pp. 121-158, 2008 .

[9] G. K. Samanta, S. C. Kumar, a Aadhi, and M. Ebrahim-Zadeh, "Ybfiber-laser-pumped, high-repetition-rate picosecond optical parametric oscillator tunable in the ultraviolet," Opt. Express, vol. 22, no. 10, p. 11476, 2014.

[10] G. H. Dieke and H. M. Crosswhite, "The Spectra of the Doubly and Triply Ionized Rare Earths,” Appl. Opt., vol. 2, no. 7, p. 675, Jul. 1963.

[11] R. T. Wegh, A. Meijerink, R.-J. Lamminmäki, and Jorma Hölsä, "Extending Dieke's diagram," J. Lumin., vol. 87-89, pp. 1002-1004, May 2000.

[12] J. E. Townsend, S. B. Poole, and D. N. Payne, "Solution-doping technique for fabrication of rare-earth-doped optical fibres," Electron. Lett., vol. 23, no. 7, p. 329, 1987.

[13] F. Moretti, N. Chiodini, M. Fasoli, L. Griguta, and A. Vedda, "Optical absorption and emission properties of Gd3+ in silica host," J. Lumin., vol. 126, no. 2, pp. 759-763, 2007.

[14] J. He, Y. Wang, S. Steigenberger, A. Macpherson, N. Chiodini, and G. Brambilla, "Intense ultraviolet photoluminescence at $314 \mathrm{~nm}$ in $\mathrm{Gd}^{\wedge} 3+-$ doped Silica," in Conference on Lasers and Electro-Optics, 2016, p. JTh2A.86

[15] H. Imai, K. Arai, H. Imagawa, H. Hosono, and Y. Abe, "Two types of oxyge-deficient centers in synthetic silica glasses," Phys. Rev. B, vol. 38, no. 17, pp. 772-775, 1988.

[16] M. Engholm and L. Norin, "Preventing photodarkening in ytterbiumdoped high power fiber lasers; correlation to the UV-transparency of the core glass.," Opt. Express, vol. 16, no. 2, pp. 1260-1268, 2008.

[17] Y. Wang, J. He, P. Barua, N. Chiodini, S. Steigenberger, M. I. M. Abdul Khudus, J. K. Sahu, M. Beresna, and G. Brambilla, "Ultraviolet photoluminescence in Gd-doped silica and phosphosilicate fibers," $A P L$ Photonics, vol. 2, no. 4, p. 46101, Apr. 2017.

[18] R. Salh, "Silicon Nanocluster in Silicon Dioxide: Cathodoluminescence, Energy Dispersive X-Ray Analysis and Infrared Spectroscopy Studies," in Crystalline Silicon - Properties and Uses, S. Basu, Ed. InTech, 2011, pp. 135-172. 